Revue des sciences de l'eau

\title{
Étude de l'impact des activités touristiques sur la qualité de l'eau et l'organisation des peuplements macrobenthiques au sein des cours d'eau de la Principauté d'Andorre Study of the mountain tourism impact on water quality and assemblage of macrobenthic populations in the Andorran rivers
}

\author{
Y. Younes, X. F. Garcia et J. Gagneur
}

Volume 15, numéro 1, 2002

URI : https://id.erudit.org/iderudit/705463ar

DOI : https://doi.org/10.7202/705463ar

Aller au sommaire du numéro

Éditeur(s)

Université du Québec - INRS-Eau, Terre et Environnement (INRS-ETE)

ISSN

0992-7158 (imprimé)

1718-8598 (numérique)

Découvrir la revue

Citer cet article

Younes, Y., Garcia, X. F. \& Gagneur, J. (2002). Étude de l'impact des activités touristiques sur la qualité de l'eau et l'organisation des peuplements macrobenthiques au sein des cours d'eau de la Principauté d'Andorre. Revue des sciences de l'eau / Journal of Water Science, 15(1), 421-424.

https://doi.org/10.7202/705463ar
Résumé de l'article

Le développement considérable de l'activité touristique hivernale et estivale entraine des perturbations au niveau des principaux cours d'eau andorrans. Il est intéressant d'étudier l'impact de ces perturbations sur des réseaux hydrographiques de haute altitude caractérisés par des conditions du milieu particulières (basse température-forte pente). Les données disponibles concernant la physicochimie et la faune des eaux andorranes sont réduites (PUIG, 1979 · PEÑA, 1983).

Treize stations ont été étudiées dans le cadre de ces travaux.

Au cours d'un cycle annuel (automne 1998 à automne 1999), huit prélèvements d'invertébrés benthiques on éte réalisés à chaque station à l'aide d'un filet Surber (vide de maille : 200 um-surface échantillonnée : $1 / 20$ ent $\mathrm{m}^{2}$ ).

Neuf paramètres physicochimiques (température, $\mathrm{pH}$, conductivité, $\mathrm{DCO}, \mathrm{DBO}_{5}$, nitrates, nitrites, ammoniaque phosphates) ont été mesurés de façon hebdomadaire à chaque station.

Le traitement des données physicochimiques par analyses discriminantes révèle l'existence d'un gradient amont-aval de dégradation de la qualité de l'eau. Parallèlement, la qualité de l'eau suit un cycle saisonnier mis en évidence par l'opposition entre campagnes d'été et d'hiver-printemps 1999, d'une part, et campagnes d'automne 1998-1999, d'autre part.

L'analyse de la répartition spatiotemporelle de la faune benthique, basée également sur des analyses discriminantes, met en évidence la disparition des taxons polluosensibles dans les stations intermédiaires et aval comme les filtreurs (Simuliidae), les prédateurs (Perlodae, Perlididae, Rhyacophilidae) et les fragmenteurs (Nemouridae, Leuctridae) et l'apparition de nouveaux taxons polluotolérants en aval (Chironomidae, Oligochètes). Ce genre de phénomène a déjà été observé dans d'autres cours d'eau de montagne soumis à des perturbations entraînant l'augmentation de la charge organique (DECAMPS et PUJOL, 1977).

Ces taxons polluotolérants comme par exemple, les Oligochètes qui prolifèrent dans la zone aval du cours d'eau principal (Gran Valira) et en aval de l'unique station d'épuration située sur l'Ariège remplacent dans la structure trophique d'autres taxons polluosensibles. Ce phénomène s'observe également au sein d'un même groupe trophique, c'est le cas des prédateurs composés des Perlidae, des Perlodidae et des Rhyacophilidae présents dans les stations de haute altitude et qui disparaissent en aval où ils sont remplacés par les Achètes (Glossiphoniidae).

Le calcul de plusieurs indices biologiques d'évaluation de la qualité des eaux (IBGN - BMWP), de richesse et de diversité spécifiques (indices de Shannon et de Margalef), de structure des peuplements (CUMMINS, 1979, 1985) met en evidence la diminution de la richesse et de la diversité spécifiques des stations amont vers les stations aval.

L'impact du développement touristique se manifeste à partir des stations de moyenne altitude. Dans ces stations, la pollution est partiellement évacuée, chaque automne, par l'eau de bonne qualité en provenance des stations, la pollution est partiellement évacuée, chaque automne, par leau de bonne qualité en p pons physicochimiques et de la composition des communaues benhes. Dans les stations aval, la pollution prer plutor un caractere chron Les résultats obtenus à partir du traitement des données physicochimiques et faunistiques mettent en évidence une dégradation de lintégrite écologique des cours d'eau (modifications physicochimiques, modifications du peuplement, diminution de la diversité). L'impact des perturbations d'origine anthropique suit un cycle dépendant de la fréquentation touristique. Au cours de ce cycle, alternent deux périodes " critiques " caractérisées par des perturbations hivernales (ski) et estivales (tourisme, randonnées) et une période de récupération " durant l'automne.

Ces travaux ont permis de tester l'hypothèse des perturbations intermédiaires du milieu (CONNELL, 1978 ; RESH, 1988 ; TOWNSEND et al., 1997) et de rechercher les seuils d'intensité des perturbations et/ou l'amplitude de ces perturbations ainsi que leur impact négatif sur la composition faunistique (EDWARD et RYKIEL, 1985; PICKETT et al., 1989; REICE et al., 1990).
Ce document est protégé par la loi sur le droit d'auteur. L’utilisation des services d'Érudit (y compris la reproduction) est assujettie à sa politique d'utilisation que vous pouvez consulter en ligne.

https://apropos.erudit.org/fr/usagers/politique-dutilisation/ 


\title{
Étude de l'impact des activités touristiques sur la qualité de l'eau et l'organisation des peuplements macrobenthiques au sein des cours d'eau de la Principauté d'Andorre
}

\author{
Study of the mountain tourism impact on water \\ quality and assemblage of macrobenthic populations \\ in the Andorran rivers
}

Y. YOUNES ${ }^{*} 1,2$, X.F. GARCIA ${ }^{2}$, J. GAGNEUR ${ }^{2}$

RÉSUMÉ

Le développement considérable de l'activité touristique hivernale et estivale entraîne des perturbations au niveau des principaux cours d'eau andorrans. Il est intéressant d'étudier I'impact de ces perturbations sur des réseaux hydrographiques de haute altitude caractérisés par des conditions du milieu particulières (basse température-forte pente). Les données disponibles concernant la physicochimie et la faune des eaux andorranes sont réduites (PUIG, 1979 ; PEÑ A, 1983).

Treize stations ont été étudiées dans le cadre de ces travaux.

Au cours d'un cycle annuel (automne 1998 à automne 1999), huit prélèvements d'invertébrés benthiques ont été réalisés à chaque station à l'aide d'un filet Surber (vide de maille : $200 \mu \mathrm{m}$-surface échantillonnée : $1 / 20^{\mathrm{e}} \mathrm{m}^{2}$ ).

Neuf paramètres physicochimiques (température, pH, conductivité, DCO, $\mathrm{DBO}_{5}$, nitrates, nitrites, ammoniaque, phosphates) ont été mesurés de façon hebdomadaire à chaque station.

Le traitement des données physicochimiques par analyses discriminantes révèle l'existence d'un gradient amont-aval de dégradation de la qualité de l'eau. Parallèlement, la qualité de l'eau suit un cycle saisonnier mis en évidence par l'opposition entre campagnes d'été et d'hiver-printemps 1999, d'une part, et campagnes d'automne 1998-1999, d'autre part.

L'analyse de la répartition spatiotemporelle de la faune benthique, basée également sur des analyses discriminantes, met en évidence la disparition des

1. Unité de recherche, Laboratoire d'environnement aquatique et aquaculture, ENSA, Toulouse, av. de l'Agrobiopole, BP 107, Auzeville Tolosane, 31326 Castanet, France.

2. Centre d'écologie des systèmes aquatiques continentaux, UMR 5576, Université Paul Sabatier, 118 , route de Narbonne, 31062 Toulouse cedex, France.

* Correspondance. E-mail : younes@aurore.cict.fr

Les commentaires seront reçus jusqu'au 31 mars 2003. 
taxons polluosensibles dans les stations intermédiaires et aval comme les filtreurs (Simuliidae), les prédateurs (Perlodae, Perlididae, Rhyacophilidae) et Ies fragmenteurs (Nemouridae, Leuctridae) et l'apparition de nouveaux taxons polluotolérants en aval (Chironomidae, Oligochètes). Ce genre de phénomène a déjà été observé dans d'autres cours d'eau de montagne soumis à des perturbations entraînant l'augmentation de la charge organique (DECAMPS et PUJOL, 1977).

Ces taxons polluotolérants comme par exemple, les Oligochètes qui prolifèrent dans la zone aval du cours d'eau principal (Gran Valira) et en aval de l'unique station d'épuration située sur l'Ariège remplacent dans la structure trophique d'autres taxons polluosensibles. Ce phénomène s'observe également au sein d'un même groupe trophique, c'est le cas des prédateurs composés des Perlidae, des Perlodidae et des Rhyacophilidae présents dans les stations de haute altitude et qui disparaissent en aval où ils sont remplacés par les Achètes (Glossiphoniidae).

Le calcul de plusieurs indices biologiques d'évaluation de la qualité des eaux (IBGN - BMWP), de richesse et de diversité spécifiques (indices de Shannon et de Margalef), de structure des peuplements (CUMMINS, 1979, 1985) met en évidence la diminution de la richesse et de la diversité spécifiques des stations amont vers les stations aval.

L'impact du développement touristique se manifeste à partir des stations de moyenne altitude. Dans ces stations, la pollution est partiellement évacuée, chaque automne, par l'eau de bonne qualité en provenance des zones amont des cours d'eau. Ce phénomène se traduit par une grande variabilité des conditions physicochimiques et de la composition des communautés benthiques. Dans les stations aval, la pollution prend plutôt un caractère chronique suite à la concentration des pollutions en provenance des différentes zones perturbées. La variabilité des conditions du milieu et des assemblages faunistiques est donc moindre.

Les résultats obtenus à partir du traitement des données physicochimiques et faunistiques mettent en évidence une dégradation de l'intégrité écologique des cours d'eau (modifications physicochimiques, modifications du peuplement, diminution de la diversité). L'impact des perturbations d'origine anthropique suit un cycle dépendant de la fréquentation touristique. Au cours de ce cycle, alternent deux périodes « critiques » caractérisées par des perturbations hivernales (ski) et estivales (tourisme, randonnées) et une période de « récupération » durant l'automne.

Ces travaux ont permis de tester l'hypothèse des perturbations intermédiaires du milieu (CONNELL, 1978 ; RESH, 1988 ; TOWNSEND et al., 1997) et de rechercher les seuils d'intensité des perturbations et/ou l'amplitude de ces perturbations ainsi que leur impact négatif sur la composition faunistique (EDWARD et RYKIEL, 1985 ; PICKETT et al., 1989 ; REICE et al., 1990).

Mots clés : cours d'eau, faune benthique, qualité de l'eau, impact touristique, Principauté d'Andorre.

The important development of tourism during winter and summer disturb the main Andorran streams. The impact of these disturbances were studied in high altitude streams characterised by particular environmental conditions (e.g. low temperature - steep slope) as little is known about water quality and associated benthic fauna in Andorran streams (PUIG, 1979; PEÑA, 1983).

Thirteen sites were seasonally studied during one year, from autumn 1998 to autumn 1999, in each site, eight macroinverbrates samples were collected 
using a Surber sampling net (mesh size $200 \mu \mathrm{m}$ - sampling area : $\mathbf{1 / 2 0 ^ { e }} \mathrm{m}^{\mathbf{2}}$ ). In addition, nine physicochemical parameters (temperature - $\mathrm{pH}$ - conductivity - DCO - $\mathrm{DBO}_{5}$ - nitrate- nitrite - ammonia - phosphate) were weekly measured in each site during the entire sampfing period.

A discriminant analysis performed on the physicochemical data reaveled and upstream-downstream gradient of the water quality, with highest disturbance in the downstream sites. From a temporal point of view, the water quality follows a seasonal cycle highlighted by the opposition between on the one hand winter-spring and summer 1999, and on the other hand autumn 1998-1999.

The spatio-temporal distribution of the benthic fauna, based on the discriminant analysis, highlighted the disappearance of polluo-sensitive taxa in intermediate and downstream sites such as filtering collectors (Simuliidae), predators (Perfidae - Perlodidae - Rhyacophilidae) and shredders (Nemouridae - Leuctridae). In these areas, sensitive taxa were replaced by polluo-tolerant ones such as Chironomidae and Oligocheta. These patterns were already observed in other mountain streams subjected to high organic disturbances (DECANTS et PUJOL, 1977).

As a consequence, polluo-tolerant taxa replace polluo-sensitives taxa with similar functionnal status. For example Oligocheta, proliferate in the downstream (Gran Valira), whereas upstream, they were only abundant on a point source pollution site located on the Ariege river. The same phenomenon was also observed for predators: high unpolluted altitude sites were characterised by stoneflies and caddisflies (Perlidae, Perlodidae, Rhyacophilidae) which were replaced downstream by leeches (Glossiphoniidae). As a consequence, the calculation of the several biological indices of water quality (I.B.G.N B.M.W.P'), and diversity descriptors (taxa richness - Shannon index - Margalef index), indicate a drastic reduction of the taxa diversity, evenness and water quality from upstream to downstream sites.

Disturbances associated with tourism development appeared in the medium altitude sites. In these sites, the water quality benefits from the good water quality from the upstream sites during high discharge periods (i.e. in autumn). This phenomena explained the observed temporal variabihty of both physicochemical conditions and benthic fauna structure. In the downstream sites, the pollution can be considered as chronic and hence, low environmental variability was observed. These results show that Andorran stream ecological integrity is low from both physicochemical diversity and population assemblages point of views. Moreover, the impact of anthropic disturbances followed an annual cycle according to tourism. activities with pollution peaks in winter and summer corresponding respectively to ski resort and hiking tourism. The only partial recovery period is represented by the autumn high drainage period.

From a theoretical point of view, these results allowed to test the intermediate disturbance hypothesis (CONNELL, 1978; RESH, 1988; TOWNSFND et al., 1997) and to search the threshold of resistence/resilience characteristics of a stream ecosystem considering stream benthic assemblages (EDWARD et RYKIEL, 1985; REICE et al., 1990; PICKETT et al., 1989).

Key-words: rivers, benthic fauna, water quality, touristic impact, Principauté d'Andorre. 


\section{RÉFÉRENCES BIBLIOGRAPHIQUES}

CONNELL J.H., 1978. Diversity in tropical rain forest and coral reefs. Science., 199, 1302-1310.

CUMMINS K.W., WILZBACH M.A., 1985. Fiels procedures for analysis on functional feeding groups of stream macroinvertebrates. Ecological Resaearch Division, Office of healt and environmental Research, $18 \mathrm{p}$.

DECAMPS H., PUJOL J.Y., 1977. Influences humaines sur le benthos d'un ruisseau de montagne dans les Pyrénées. Bull. Ecol., 8 (3), 349-358.

EDWARD J., RYKIEL J.R., 1985. Towards a definition of ecological disturbance. Aust. J. Ecol., 10, 361-365.

PENA G., 1983. Factores que determinan la distribucion de los simulidos y quironomidos (Diptera) de la red hidrografica andorrana. Actas del primer congreso espagnol de limnologia, 197-205.

PICKETT S.T.A., KOLASA J., ARMESTO J.J., COLLINS S.L., 1989. The ecological concept of disturbance and its expression at various hierarchical levels. Oikos., $54,129-136$.

PUIG M.A., 1980. Contribucio a l'estudi de l'ecologia comparada dels plecopters i efemeropters d'Andorra. Bull. Inst. Cat. Hist. Nat., 45, 155 p.

REICE S.R., WISSMAR R.C., NAIMAN R.J., 1990. Disturbance regimes, resilience, and recovery of animal communities and habitats in lotic ecosystems. Environ. Manage., 14 (5), 647-659.

RESH V.H., BROWN A.V., COVICH A.P., GURTZ M.E., MINSHALL H.W.L.G.W., REICE S.R., SHELDON A.L., WALLACE J.B., WISSMAR R.C., 1988. The role of disturbance in stream ecology. J.N.Am. Benthol. Soc., 7, 433-455.

TOWNSEND C.R., SCARSBROOK M.R., DOLEDEC S., 1997. The intermediate distrubance hypothesis, refugia, and biodiversity in streams. Limnol. Oceanogr., 42 (5), 938-949. 